\title{
ALGEBRAIC BARTH-LEFSCHETZ THEOREMS
}

\author{
LUCIAN BĂDESCU
}

\section{Introduction}

We shall work over a fixed algebraically closed field $k$ of arbitrary characteristic. By an algebraic variety over $k$ we shall mean a reduced algebraic scheme over $k$. Fix a positive integer $n$ and $e=\left(e_{0}, e_{1}, \ldots, e_{n}\right)$ a system of $n+1$ weights (i.e. $n+1$ positive integers $e_{0}, e_{1}, \ldots, e_{n}$ ). If $k\left[T_{0}, T_{1}, \ldots, T_{n}\right]$ is the polynomial $k$-algebra in $n+1$ variables, graded by the conditions $\operatorname{deg}\left(T_{i}\right)=e_{i}, i=0,1, \ldots$, $n$, denote by $\mathbf{P}^{n}(e)=\operatorname{Proj}\left(k\left[T_{0}, T_{1}, \ldots, T_{n}\right]\right)$ the $n$-dimensional weighted projective space over $k$ of weights $e$. We refer the reader to [3] for the basic properties of weighted projective spaces. According to Zariski ([22], see also [16], [15]), if $Y$ is a closed subscheme of an algebraic variety $X$, one can define the ring $K\left(\hat{X}_{/ Y}\right)$ of formal rational functions of $X$ along $Y$. Then $K\left(\hat{X}_{/ Y}\right)$ is a $k$-algebra, and there is a canonical map of $k$-algebras $K(X) \rightarrow K\left(\hat{X}_{/ Y}\right)$, where $K(X)$ is the usual ring of rational functions of $X(K(X)$ is a field if $X$ is irreducible). According to [16], $Y$ is said to be $\mathbf{G}_{3}$ in $X$ if this map is an isomorphism. Let $X$ be an arbitrary algebraic scheme over $k$, and let $d \geq 0$ be a non-negative integer. Then $X$ is said to be $d$-connected if every irreducible component of $X$ is of dimension $\geq d+1$ and if $X \backslash W$ is connected for every closed subscheme $W$ of $X$ of dimension $<d$. For example, $X$ is 0 -connected if $X$ is connected and of dimension $\geq 1$; an irreducible algebraic variety $X$ of dimension $n \geq 1$ is always $(n-1)$-connected.

Then the main result of this paper is the following.

Theorem (0.1). Let $f: X \rightarrow \mathbf{P}^{n}(e) \times \mathbf{P}^{n}(e)$ be a finite morphism from a $d$-connected algebraic variety $X$ such that $d \geq n$. Then $f^{-1}(\Delta)$ is $(d-n)$-connected, where $\Delta$ is the diagonal of $\mathbf{P}^{n}(e) \times \mathbf{P}^{n}(e)$. Moreover, $f^{-1}(\Delta) \backslash W$ is $\mathbf{G}_{3}$ in $X \backslash W$ for every closed subscheme $W$ of $f^{-1}(\Delta)$ of dimension $<d-n$.

In the case of ordinary projective spaces (i.e. when $e_{i}=1$ for every $i=0$, $1, \ldots, n)$ the first statement of Theorem $(0.1)$ is well known in the literature as

\footnotetext{
Received October 25, 1994.
} 
the Fulton-Hansen connectedness theorem (see [5], or also [6], or [12]). As far as I know the last part of Theorem (0.1) is new even for ordinary projective spaces. This latter fact has some interesting consequences, namely the following two theorems.

THeORem (0.2). Let $Y$ be a closed irreducible subvariety of $\mathbf{P}^{n}(e)$ of dimension $>\frac{n}{2}$. Then $\Delta_{Y} \backslash W$ is $\mathbf{G}_{3}$ in $Y \times Y \backslash W$ for every closed subscheme $W$ of the diagonal $\Delta_{Y}$ of $Y \times Y$ such that $\operatorname{dim}(W)<2 \operatorname{dim}(Y)-n-1$. If moreover $Y$ has (locally) the $\mathbf{S}_{2}$ property of Serre (e.g. if $Y$ is normal), then for every vector bundle $E$ on $Y \times Y$ the natural map

$$
H^{0}(Y \times Y, E) \rightarrow H^{0}(\widehat{Y \times Y}, \hat{E})
$$

is an isomorphism, where $\hat{E}$ is the formal completion of $E$ along $\Delta_{Y}$, and $\widehat{Y \times Y}=$ $Y \times Y_{\Delta_{\Delta_{Y}}}$ In other words, the weak Grothendieck-Lefschetz condition $\operatorname{Lef}\left(Y \times Y, \Delta_{Y}\right)$ holds (see [9]).

An immediate consequence of Theorem (0.2) via a result of Speiser (see [21], or also Theorem (1.11) below) is the following.

Theorem (0.3). Let $Y$ be a closed irreducible subvariety of $\mathbf{P}^{n}(e)$ of dimension $>\frac{n}{2}$ which is (locally) $\mathbf{S}_{2}$. Then every stratified vector bundle on $Y$ is trivial.

For the definition of stratified vector bundles see [10], or also [20]. This definition and the basic properties of stratified vector bundles will also be briefly recalled in the first section. In some special cases Theorem (0.3) and the last part of Theorem (0.2) were known before. Specifically, if $Y$ is a local complete intersection in $\mathbf{P}^{n}$ over a field $k$ of characteristic zero, Theorem (0.3) and the last part of Theorem (0.2) were proved by Ogus in [18]. Note that when $\operatorname{char}(k)=0$ the concept of stratified vector bundle is the same as the one of vector bundle with integrable connection (see [18]). If $\operatorname{char}(k)>0$ and $Y$ is an irreducible locally Cohen-Macaulay of $\mathbf{P}^{n}$ these results were proved by Speiser in [21]. Note that the methods of Ogus (in characteristic 0) or those of Speiser (in positive characteristic) are completely different from the methods used in this paper. Our approach (which is based on results of Hironaka-Matsumura [16] and of Faltings [4] involving formal rational functions) offer therefore not only characteristic free proofs but also more general results.

A first version of this paper was written during my visit at the Universities 
of Pisa (December 1993) and Ferrara (January 1994). I am grateful to Fabrizio Catanese and Alexandru Lascu for their kind invitation and excellent atmosphere I found there. I also want to thank G. Chiriacescu who read some parts of the paper and made useful remarks and suggestions.

\section{Background material}

In this section we gather together some known results we are going to use in this paper. Let $X$ be an algebraic variety, and let $X_{1}, \ldots, X_{m}$ be the irreducible components of $X$. Fix $d \geq 0$ a non-negative integer.

Definition (1.1) ([13], [12]). A sequence $Z_{0}, Z_{1}, \ldots, Z_{n}$ of (not necessarily mutually distinct) irreducible components of $X$ is called a $d$-join within $X$ if $\operatorname{dim}\left(Z_{i}\right) \geq d+1$ for every $i=0,1, \ldots, n$ and if $\operatorname{dim}\left(Z_{j-1} \cap Z_{j}\right) \geq d$ for every $j=2, \ldots, n$.

The following elementary fact will be useful.

Proposition (1.2) (Hartshorne [13], or also [12]). An algebraic variety $X$ is $d$-connected if and only if $X=Z_{0} \cup Z_{1} \cup \ldots \cup Z_{n}$ for some $d$-join $Z_{0}, Z_{1}, \ldots, Z_{n}$ within $X$.

Theorem (1.3) (Grothendieck). Let $X$ be a $d$-connected algebraic variety over $k$, and let $f: X \rightarrow \operatorname{Proj}(S)$ be a finite morphism, where $S$ is a finitely generated graded $k$-algebra. Let $t_{1}, \ldots, t_{r} \in S_{+}$be homogeneous elements of positive degrees. If $d \geq r$ then $f^{-1}\left(V_{+}\left(t_{1}, \ldots, t_{r}\right)\right)$ is $(d-r)$-connected. Moreover, if $X$ is irreducible and $\operatorname{dim}(X) \geq r$ then $f^{-1}\left(V_{+}\left(t_{1}, \ldots, t_{r}\right)\right)$ is non-empty.

Remark. Theorem (1.3) can be found (in a slightly different formulation) in [9] éxposé XIII. In the appendix another proof based on the so-called HartshorneLichtenbaum theorem can be found.

Theorem (1.4) (Hironaka-Matsumura [16]). Let $f: X^{\prime} \rightarrow X$ be a proper surjective morphism of algebraic varieties, with $X$ irreducible and such that every irreducible component of $X^{\prime}$ dominates $X$. Let $Y$ be a closed subscheme of $X$ and set $Y^{\prime}:=$ $f^{-1}(Y)$. Then there is a canonical isomorphism of $k$-algebras

$$
K\left(\hat{X}_{/ Y^{\prime}}^{\prime}\right) \cong\left[K\left(\hat{X}_{/ Y}\right) \bigotimes_{K(X)} K\left(X^{\prime}\right)\right]_{0},
$$


where $K\left(X^{\prime}\right)$ is the ring of rational functions of $X^{\prime}, K\left(\hat{X}_{/ Y^{\prime}}\right)$ is the ring of formal rational functions of $X^{\prime}$ along $Y^{\prime}$, and $[A]_{0}$ denotes the total ring of fractions of a commutative ring $A$.

The next result will play a crucial role in the proof of Theorem $(0.1)$.

Theorem (1.5) (Faltings [4]). Let $X$ be an irreducible closed subvariety of $\mathbf{P}^{n}$, and let $L$ be a linear subspace of $\mathbf{P}^{n}$ of codimension $r$ such that $r<\operatorname{dim}(X)$. Then $X \cap L \backslash W$ is $\mathbf{G}_{3}$ in $X \backslash W$ for every closed subvariety $W$ of $X \cap L$ such that $\operatorname{dim}(W)<\operatorname{dim}(X)-r-1(\operatorname{dim}(W)=-1$ if $W=\emptyset)$.

Remark. Theorem (1.5) is a special case of a result of Faltings [4]. In the case when $X$ and $L$ have a proper intersection, this result was proved earlier by Hironaka and Matsumura [16] (in which case the proof is much more elementary).

Proposition (1.6) ([16]). Let $X$ be an irreducible algebraic variety, and let $Y$ be a closed subscheme of $X$. Let $u: X^{\prime} \rightarrow X$ be the normalization morphism. Then $K\left(\hat{X}_{/ Y}\right)$ is a field if and only if $u^{-1}(Y)$ is connected.

The next proposition is well known for the rings of usual rational functions, and should be known in general, but we have no reference for it (therefore we include a proof).

Proposition (1.7). Let $X$ be a quasi-projective variety having the irreducible components $X_{1}, \ldots, X_{m}$ (with the reduced structure), and let $Y$ be a closed subscheme of $X$, such that $Y_{i}:=Y \cap X_{i} \neq \emptyset$ for every $i=1, \ldots, m$. Then there is a canonical isomorphism of $k$-algebras

$$
K\left(\hat{X}_{/ Y}\right) \cong K\left(\hat{X}_{1 / Y_{1}}\right) \times \cdots \times K\left(\hat{X}_{m_{/ Y_{m}}}\right) .
$$

Proof. We shall first prove Proposition (1.7) in the case when $X=$ $\operatorname{Spec}(A)$ is affine, with $A$ a reduced finitely generated $k$-algebra. Set $\operatorname{Ass}(A)=$ $\left\{p_{1}, \ldots, p_{m}\right\}$, where every $p_{i}$ is a minimal prime ideal of $A$ such that $X_{i}=V\left(p_{i}\right)$, $i=1, \ldots, m$ and

$$
p_{1} \cap \cdots \cap p_{m}=(0)
$$

Then $Y=V(I)$, with $I$ an ideal of $A$. The hypotheses imply that $I_{i}:=I\left(A / p_{i}\right) \neq$ $A / p_{i}$ for every $i=1, \ldots, m$. Let $\hat{A}$ be the $I$-adic completion of $A$. Then $\hat{X}_{/ Y}=$ 
$\operatorname{Spf}(\hat{A})$, and by [16], (1.1) we have

$$
K\left(\hat{X}_{/ Y}\right)=[\hat{A}]_{0},
$$

where, as above, $[\hat{A}]_{0}$ denotes the total ring of fractions of $\hat{A}$. Set $q_{\imath}=p_{i} \hat{A}, i=$ $1, \ldots, m$. Taking into account that the homomorphism $A \rightarrow \hat{A}$ is flat, (1.7.1) yields

$$
q_{1} \cap \cdots \cap q_{m}=0,
$$

which implies

$$
q_{1}[\hat{A}]_{0} \cap \cdots \cap q_{m}[\hat{A}]_{0}=(0) .
$$

If we denote by $S$ (resp. by $\hat{S}$ ) the multiplicative system of all non-zero divizors of $A$ (resp. of $\hat{A}$ ), then $p_{i} S^{-1} A+p_{j} S^{-1} A=S^{-1} A$ for every $i \neq j$, which implies $p_{i} S^{-1} \hat{A}+p_{j} S^{-1} \hat{A}=S^{-1} \hat{A}$ for every $i \neq j$, or else, $q_{i} S^{-1} \hat{A}+q_{j} S^{-1} \hat{A}=S^{-1} \hat{A}$ for every $i \neq j$. Since $S \subset \widehat{S}$ (because $A \rightarrow \hat{A}$ is flat), we therefore get:

$$
q_{i}[\hat{A}]_{0}+q_{j}[\hat{A}]_{0}=[\hat{A}]_{0} \text { for } i \neq j .
$$

Then (1.7.2), (1.7.3) and (1.7.4) together with the Chinese Remainder Theorem imply

$$
K\left(\hat{X}_{/ Y}\right) \cong[\hat{A}]_{0} / q_{1}[\hat{A}]_{0} \times \cdots \times[\hat{A}]_{0} / q_{m}[\hat{A}]_{0} .
$$

To prove the proposition in case $X$ is affine it will be sufficient to show that

$$
[\hat{A}]_{0} / q_{\imath}[\hat{A}]_{0} \cong K\left(\hat{X}_{i / Y_{i}}\right) \text { for every } i=1, \ldots, m \text {. }
$$

Since $K\left(\hat{X}_{i / Y_{i}}\right) \cong\left[\hat{A} / q_{i}\right]_{0}([16],(1.1))$, then (1.7.6) is equivalent to

$$
\hat{S}^{-1}\left(\hat{A} / q_{i}\right)=\left[\hat{A} / q_{i}\right]_{0} \text { for every } i=1, \ldots, m .
$$

To prove (1.7.7), we first show that for every fixed $i$ the ring $\hat{A} / q_{i}$ is reduced. To see this, observe that $\hat{A} / q_{i}$ is the $I$-adic completion of $A / p_{i}$. So, we have to prove that if $B$ is a reduced, finitely generated $k$-algebra, and $J \neq B$ is an ideal of $B$ then the $J$-adic completion $B^{*}$ of $B$ is also reduced. To show this, it will be sufficient to check that for every maximal ideal $m^{*}$ of $B^{*}$, the localization $B^{*}{ }_{m^{*}}$ is reduced. Since $B^{*}$ is $J B^{*}$-adically complete, $J B^{*} \subset m^{*}$. Since $B^{*} / J^{n} B^{*}$ $\cong B / J^{n}$, it follows that for every $n \geq 1, B_{m^{*}}^{*} / J^{n} B_{m^{*}} \cong B_{m} / J^{n} B_{m}$, where $m:=m^{*} \cap B$. From the latter isomorphism we infer that for every $n \geq 1$, $B_{m^{*}}^{*} / m^{* n} B_{m^{*}}^{*} \cong B_{m} / m^{n} B_{m}$. Passing to the inverse limits, we get $\widehat{B}_{m^{*}}^{*} \cong \hat{B}_{m}$ (the completions with respect to the maximal ideals $m^{*}$ and $m$ respectively). Now, use a theorem of Chevalley (see [19], IV-4) to show that the latter ring is reduced. Finally, since $\widehat{B}_{m^{*}}^{*}$ is reduced, $B_{m^{*}}^{*}$ is also reduced, and we are through. 
So, we have seen that $\hat{A} / q_{i}$ is reduced. In particular, we get

$$
q_{\imath}=p_{i 1} \cap \cdots \cap p_{i n_{i}},
$$

where $\operatorname{Ass}\left(\hat{A} / q_{i}\right)=\left\{p_{i 1}, \ldots, p_{i n_{i}}\right\}$.

Then by [19], IV $-4, \operatorname{Ass}(\bar{A})=\left\{p_{11}, \ldots, p_{1 n_{1}}, \ldots, p_{m 1}, \ldots, p_{m n_{m}}\right\}$. Moreover, all the $p_{i j}$ 's are minimal prime ideals of $\hat{A}$.

The inclusion $\hat{S}^{-1}\left(\hat{A} / q_{i}\right) \subset\left[\hat{A} / q_{i}\right]_{0}$ being clear, it will be sufficient to show that every non-zero divisor $\bar{a}:=a\left(\bmod q_{i}\right)$ of $\hat{A} / q_{i}$ is the class (modulo $q_{i}$ ) of an element of $\hat{S}$ (i.e. $\hat{a}=\hat{b}$ with $b$ a non-zero divisor of $\hat{A}$, or else, $b \notin p_{i}$, for all $i$ and $j$ ). Take for example $i=1$. Since $\hat{a}$ is a non-zero divisor in $\bar{A} / q_{1}, a \notin\left\{p_{11}\right.$ $\left.\cup \cdots \cup p_{1 n_{1}}\right\}$. Set $\Lambda:=\left\{(i, j) / a \in p_{i j}\right\}$. If $\Lambda$ is empty we have nothing to prove. Suppose therefore $\Lambda \neq \emptyset$. Then set $J:=\bigcap_{(i, j) \in \Lambda} p_{i j}$ and $J^{\prime}:=\bigcap_{(i, j) \in \Lambda} p_{i j}$. Since all the ideals $p_{i j}$ are prime, by elementary general facts about prime ideals (see e.g. [19], I-2), there is an element $u \in J \backslash J^{\prime}$. In other words, $u \in p_{i}$, if and only if $a \notin$ $p_{i j}$. This shows that $a+u \notin p_{i j}$ for every $i=1, \ldots, m, j=1, \ldots, n_{\imath}$. On the other hand, since $a \notin p_{1 j}$ for every $j=1, \ldots, n_{1}$, whence $u \in p_{11} \cap \cdots \cap p_{1 n_{1}}=$ $q_{1}$ : Therefore $\hat{a}=\hat{b}$, with $b=a+u$, and $b$ not a zero divisor in $\hat{A}$. Therefore Proposition (1.7) is proved if $X$ is affine.

If $X$ is not affine, fix a finite set $A$ of closed points of $Y$ such that $A \cap Y_{i} \neq$ $\emptyset$ for every $i=1, \ldots, m$. Since $X$ is quasi-projective, we can find an affine cover $\left\{U_{\alpha}\right\}_{\alpha}$ of $X$ such that for every $\alpha, A \subset U_{\alpha}$. Since for every $\alpha$ and $\beta, U_{\alpha \beta}:=$ $U_{\alpha}$

$\cap U_{\beta}$ is also affine and contains $A$, we know the proposition for $U_{\alpha}$ and for $U_{\alpha \beta}$. Then everything follows from the statement in the affine case (already proved) and from the exact diagram (see [16] for details):

$$
K\left(\hat{X}_{/ Y}\right) \rightarrow \prod_{\alpha} K\left(\hat{U}_{\alpha / Y \cap U_{\alpha}}\right) \rightrightarrows \prod_{\alpha, \beta} K\left(\hat{U}_{\alpha \beta / Y \cap U_{\alpha \beta}}\right),
$$

which reduces the verification to the affine case.

Q.E.D.

Corollary (1.8). In the hypotheses of Proposition (1.7), if $Y_{\imath}$ is $\mathbf{G}_{3}$ in $X_{i}$ for every $i=1, \ldots, m$, then $Y$ is also $\mathbf{G}_{3}$ in $X$.

Proof. Direct consequence of Proposition (1.7) and of the well known isomorphism

$$
K(X)=K\left(X_{1}\right) \times \cdots \times K\left(X_{m}\right)
$$


Corollary (1.8) allows one to generalize the result of Faltings stated above (Theorem (1.5)):

Corollary (1.9). Let $X$ be a d-connected closed subvariety of $\mathbf{P}^{n}$, and let $L$ be a linear subspace of $\mathbf{P}^{n}$ of codimension $r$ such that $r<d$. Then $X \cap L \backslash W$ is $\mathbf{G}_{3}$ in $X \backslash W$ for every closed subscheme $W$ of $X \cap L$ such that $\operatorname{dim}(W)<d-r$.

Proof. Let $X_{1}, \ldots, X_{m}$ be the irreducible components of $X$. Since $X$ is $d$-connected, $\operatorname{dim}\left(X_{i}\right) \geq d+1$, and in particular, $r<\operatorname{dim}\left(X_{i}\right)$ for every $i=$ $1, \ldots . m$. By Theorem (1.5), $X_{i} \cap L \backslash W$ is $\mathbf{G}_{3}$ in $X_{i} \backslash W$, i.e. $\left.K\left(X_{i} \backslash W\right)\right) \cong$ $K\left({\widehat{X} i W_{/ X_{i} \cap L \backslash W}}\right)$ for every $i=1, \ldots, m$ and for every closed subscheme $W$ of $X \cap L$ such that $\operatorname{dim}(W)<d-r$. On the other hand, since the irreducible components of $X \backslash W$ are $X_{i} \backslash W, i=1, \ldots, m$ everything follows from Corollary (1.8).

Q.E.D.

(1.10) Now we recall briefly some definitions and basic facts from Grothendieck's theory of stratified vector bundles and the descent theory of faithfully flat morphisms (see [10], [8], or also [21]). Let $Y$ be an algebraic variety over $k$. Consider the products and projections:

$$
Y \stackrel{p_{1}}{\longleftarrow} Y \times Y \stackrel{p_{2}}{\longrightarrow} Y
$$

and

$$
\begin{aligned}
& Y \times Y \stackrel{p_{21}}{\longleftarrow} Y \times Y \times Y \stackrel{p_{31}}{\longrightarrow} Y \times Y \\
& Y \times Y
\end{aligned}
$$

Denote by $\Delta=\Delta_{Y}$ the diagonal of $Y \times Y$, and let $\Delta^{\prime}$ be the diagonal subscheme of $Y \times Y \times Y$. For every $r \geq 0$ denote by $\Delta_{r}$ (resp. $\Delta_{r}^{\prime}$ ) the $r$-th infinitesimal neighbourhood of $\Delta$ in $Y \times Y$ (resp. of $\Delta^{\prime}$ ). Then from the previous diagrams we get projections

$$
Y \stackrel{q_{1}^{r}}{\longleftarrow} \Delta_{r} \stackrel{q_{2}^{r}}{\longrightarrow} Y
$$

and

$$
\begin{aligned}
& \Delta_{r} \stackrel{q_{21}^{r}}{\stackrel{q_{31}^{\prime}}{\longrightarrow}} \Delta_{r}^{\prime} . \\
& \downarrow p_{32}^{r} \\
& \Delta r
\end{aligned}
$$

Let $F$ be a vector bundle on $Y$. By descent data on $F$ we mean an isomorph- 
ism

$$
\varphi: p_{1}^{*}(F) \rightarrow p_{2}^{*}(F)
$$

over $Y \times Y$ such that the cocycle condition

$$
p_{31}^{*}(\varphi)=p_{32}^{*}(\varphi) \circ p_{21}^{*}(\varphi)
$$

holds over $Y \times Y \times Y$.

From Grothendieck's faithfully flat descent theory (see [8], VIII) applied to the structural morphism $p: Y \rightarrow \operatorname{Spec}(k)$ we know that descent data hold for $F$ if and only if $F$ is the pull-back via $p$ of a vector bundle over $\operatorname{Spec}(k)$, i.e. if and only if $F$ is a trivial vector bundle. This result is a very useful general criterion for a vector bundle to be trivial.

A stratification on $F$ is a compatible system of isomorphisms

$$
\varphi_{r}:\left(q_{1}^{r}\right)^{*}(F) \rightarrow\left(q_{2}^{r}\right)^{*}(F)
$$

over $\Delta_{r}$ for all $r \geq 0$ such that $\varphi_{0}=$ id and the cocycle condition

$$
\left(q_{31}^{r}\right) *\left(\varphi_{r}\right)=\left(q_{32}^{r}\right)^{*}\left(\varphi_{r}\right) \circ\left(q_{21}^{r}\right)^{*}\left(\varphi_{r}\right)
$$

holds on $\Delta_{r}^{\prime}$.

A stratified vector bundle on $Y$ is a vector bundle $F$ with a stratification on it. In other words, giving a stratification on $F$ is the same as giving "formal descent data" on $F$. That is to say, if $X$ is the formal completion of $Y \times Y$ along $\Delta$, and if $X^{\prime}$ is the formal completion of $Y \times Y \times Y$ along $\Delta^{\prime}$, then we get projections

$$
Y \stackrel{q_{1}}{\longleftarrow} X \stackrel{q_{2}}{\longrightarrow} Y
$$

and

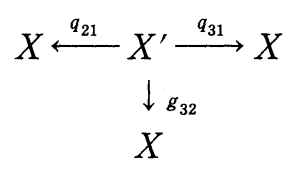

and "formal descent data" on $F$ consist of an isomorphism

$$
\phi: q_{1}^{*}(F) \rightarrow q_{2}^{*}(F)
$$

on $X$ together with the cocycle condition

$$
q_{31}^{*}(\phi)=q_{32}^{*}(\phi) \circ q_{21}^{*}(\phi)
$$

on $X^{\prime}$. In characteristic zero giving a stratification on $F$ is the same as giving an integrable connection on $F$ (see [10], [18]). The approach of vector bundles en- 
dowed with integrable connections and the De Rham cohomology associated to them was taken up by Ogus to give algebraic proofs of Barth's theorems (see [18]). On the other hand, Gieseker and Speiser provided further methods to study stratifications in positive characteristic (see [7], [21]). The relationship between stratified vector bundles and descent data is given by the following result of Speiser:

Theorem (1.11) (Speiser). Let $Y$ be an irreducible algebraic variety over $k$ for which the diagonal $\Delta$ of $Y \times Y$ satisfies the weak Grothendieck-Lefschetz condition Lef $(Y \times Y, \Delta)$. Then giving a stratification on a vector bundle $F$ on $Y$ is equivalent to giving descent data on $F$. In particular, if $\operatorname{Lef}(Y \times Y, \Delta)$ holds, then every stratified vector bundle on $Y$ is trivial.

In fact Speiser proved in [21] the first part of Theorem (1.11); the last part follows from the first one and from Grothendieck's faithfully flat descent theory [8], VIII.

\section{Proof of Theorem $(0.1)$}

To prove the connectivity part of Theorem $(0.1)$ we use the same main ideas of the proof of Fulton-Hansen connectivity theorem given in [3] or in [6]. First we shall need the following more general version of Theorem (1.3) of Grothendieck.

Proposition (2.1). Let $S$ be a finitely generated graded $k$-algebra, $t_{1}, \ldots, t_{r} \in$ $S_{+}$homogeneous elements of positive degrees, and $U$ a Zariski open subset of $\operatorname{Proj}(S)$ containing $L:=V_{+}\left(t_{1}, \ldots, t_{r}\right)$. Let $f: X \rightarrow U$ be a finite morphism, with $X$ a $d$-connected algebraic variety over $k$. If $d \geq r$ then $f^{-1}(L)$ is $(d-r)$-connected.

Proof. First we shall prove the proposition in the case when $X$ is irreducible. Then $X$ is $(\operatorname{dim}(X)-1)$-connected. Therefore in this case the hypothesis reads $\operatorname{dim}(X)>r$. By passing to the normalization we may assume that $X$ is normal. Let $Z^{\prime}$ be the closure of $X^{\prime}:=f(X)$ in $P:=\operatorname{Proj}(S)$, and let $g: Z \rightarrow Z^{\prime}$ be the normalization of $Z^{\prime}$ in the field $K(X)$ of rational function of $X$ (which makes sense because the dominant morphism $X \rightarrow Z^{\prime}$ yields the finite field extension $K\left(Z^{\prime}\right)=$ $\left.K\left(X^{\prime}\right) \rightarrow K(Z)\right)$. Then we get a commutative diagram of the form

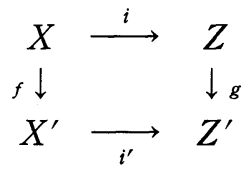


in which $i$ and $i^{\prime}$ are open immersions ( $i$ is an open immersion because $X$ is normal), and $g$ is a finite morphism. Since $L \subset U$ and $X^{\prime} \subset U$, then $X^{\prime} \cap L=$ $Z^{\prime} \cap L$, whence $f^{-1}(L)=g^{-1}(L)$. Then Proposition (2.1) follows (in case when $X$ is irreducible) from Theorem (1.3) applied to the composition of the finite morphism $g: Z \rightarrow Z^{\prime}$ followed by the closed immersion $Z^{\prime} \subset P$.

Assume now $X$ reducible. In this case we shall first prove the proposition in the special case $d=r$. Then we simply have to show that $Y:=f^{-1}(L)$ is connected and non-empty. Let $X_{1}, \ldots, X_{m}$ be the irreducible components of $X$ (all of dimension $\geq d+1=r+1$ since $X$ is $d$-connected). If we set $f_{i}:=f / X_{i}$ then by what we have proved so far we know that $Y_{i}:=f_{i}^{-1}(L)$ is connected and nonempty for every $i=1, \ldots, m$. Clearly, $Y=Y_{1} \cup \ldots \cup Y_{m}$. To prove that $Y$ is connected it will be then sufficient to show that we can reorder the components $X_{1}, \ldots, X_{m}$ (possibly with repetitions, by increasing $m$ if necessary) in such a way that $Y_{i-1} \cap Y_{i} \neq \emptyset$ for every $i=2, \ldots, m$. To do that we use Proposition (1.2) to get a reordering (possibly with repetitions) $X_{1}, \ldots, X_{m}$ such that $\operatorname{dim}\left(X_{i-1} \cap\right.$ $\left.X_{i}\right) \geq d$ for every $i=2, \ldots, m$. Therefore for every $i=2, \ldots, m$ there is an irreducible component $Z_{i}$ of $X_{i-1} \cap X_{i}$ such that $\operatorname{dim}\left(Z_{i}\right) \geq d$. Since $d \geq r$, then apply the last part of Theorem (1.3) to the $u_{i}:=f / Z_{i}: Z_{i} \rightarrow P$ to deduce that $u_{i}^{-1}(L) \neq \varnothing$ for every $i=2, \ldots, m$. Since $u_{i}^{-1}(L) \subset Y_{i-1} \cap Y_{i}$, it follows that $Y_{i-1} \cap Y_{i} \neq \emptyset$ for every $i=2, \ldots, m$, as desired.

Therefore Proposition (2.1) is proved in case $d=r$. The case $d>r$ can be reduced to the case $d=r$ as follows. Let $W$ be a closed subscheme of $Y$ of dimension $<d-r$. Then $f(W)$ is a closed subscheme of $Y$ of dimension $<d-r$. Pick a sufficiently large integer $a>0$ such that $\mathscr{O}_{P}(a)$ is a very ample line bundle on $P$ such that $S_{a} \cong H^{0}\left(P, \mathscr{O}_{P}(a)\right)$. The existence of such an $a$ comes from the fact that $S$ is a finitely generated graded $k$-algebra. Let $t_{r+1}, \ldots, t_{d} \in S_{a}=H^{0}(P$, $\mathfrak{O}_{P}(a)$ ) be $d-r$ general homogeneous elements of degree $a$ of $S$. Since $\operatorname{dim}(f(W))<d-r$ and $t_{r+1}, \ldots, t_{d}$ are general, we infer that $f(W) \cap V_{+}\left(t_{r+1}\right.$, $\left.\ldots, t_{d}\right)=\emptyset$, whence $W \cap Y^{\prime}=\emptyset$, where $Y^{\prime}:=f^{-1}\left(V_{+}\left(t, \ldots, t_{d}\right)\right)$. By what we have already seen before, $Y^{\prime}$ is connected and non-empty. Moreover, let $Z$ be an arbitrary irreducible component of $Y$. Since $X$ is $d$-connected and $Y=$ $f^{-1}\left(V_{+}\left(t_{1}, \ldots, t_{r}\right)\right), \operatorname{dim}(Z)>d-r$, and hence $\operatorname{dim}(f(Z))>d-r$ because $f$ is finite. In particular, $f(Z)$ meets $V_{+}\left(t_{r+1}, \ldots, t_{d}\right)$, or else, $Z$ meets $Y^{\prime}$. Suppose that $Y \backslash W$ is disconnected; then $Y^{\prime}$ is also disconnected because $Y^{\prime}$ meets every irreducible component of $Y$ and $Y^{\prime} \cap W=\emptyset$, a contradiction.

Q.E.D.

(2.2) We shall show that a construction used by Deligne (see [3], or also [6]) to simplify the proof of Fulton-Hansen connectedness theorem can easily be gener- 
alized to weighted projective spaces. Having the system $e=\left(e_{0}, e_{1}, \ldots, e_{n}\right)$ of weights fixed, consider the weighted projective space

$$
\mathbf{P}^{2 n+1}(e, e)=\operatorname{Proj}\left(k\left[T_{0}, \ldots, T_{n} ; U_{0}, \ldots, U_{n}\right]\right),
$$

where $T_{0}, \ldots, T_{n}, U_{0}, \ldots, U_{n}$ are $2 n+2$ indeterminates over $k$ such that $\operatorname{deg}\left(T_{i}\right)=\operatorname{deg}\left(U_{i}\right)=e_{i}$ for every $i=0,1, \ldots, n$. Consider the closed subschemes

$$
L_{1}=V_{+}\left(T_{0}, \ldots, T_{n}\right) \text { and } L_{2}=V_{+}\left(U_{0}, \ldots, U_{n}\right)
$$

of $P:=\mathbf{P}^{2 n+1}(e, e)$. Then $L_{1} \cap L_{2}=\emptyset$. Set $U:=P \backslash\left(L_{1} \cup L_{2}\right)$. Since $T_{i}-U_{i}$ is a homogeneous element of degree $e_{i}$, it makes sense to consider also the closed subscheme $H:=V_{+}\left(T_{0}-U_{0}, \ldots, T_{n}-U_{n}\right)$ of $P$. Clearly, $H \subset U$. The two natural inclusions $k\left[T_{0}, \ldots, T_{n}\right] \subset k\left[T_{0}, \ldots, T_{n} ; U_{0}, \ldots, U_{n}\right]$ and $k\left[U_{0}, \ldots, U_{n}\right] \subset$ $k\left[T_{0}, \ldots, T_{n} ; U_{0}, \ldots, U_{n}\right]$ yield two rational maps $g_{i}: \mathbf{P}^{2 n-1}(e, e) \cdots \mathbf{P}^{n}(e), i=$ 1,2 , which give rise to rational map

$$
g: \mathbf{P}^{2 n+1}(e, e) \cdots \rightarrow \mathbf{P}^{n}(e) \times \mathbf{P}^{n}(e) .
$$

Then $g$ is defined precisely in the open subset $U$ of $\mathbf{P}^{2 n+1}(e, e)$. Alternatively, if we interpret $\mathbf{P}^{n}(e)$ as the geometric quotient $\left(k^{n+1} \backslash\{0\}\right) / \mathbf{G}_{m}$ (where the action of the multiplicative group $\mathbf{G}_{m}=k \backslash\{0\}$ on $k^{n+1} \backslash\{0\}$ is given by $\lambda\left(t_{0}, \ldots, t_{n}\right):=$ $\left(\lambda^{e_{0}} t_{0}, \ldots, \lambda^{e_{n}} t_{n}\right)$, with $\lambda \in \mathbf{G}_{m}$ and $\left.\left(t_{0}, \ldots, t_{n}\right) \in k^{n+1} \backslash\{0\}\right)$, then the map $g$ is defined by

$$
g\left(\left[t_{0}, \ldots, t_{n} ; u_{0}, \ldots, u_{n}\right]\right)=\left(\left[t_{0}, \ldots, t_{n}\right],\left[u_{0}, \ldots, u_{n}\right]\right) .
$$

It is clear that $g / H$ defines an isomorphism $H \cong \Delta$. Consider the commutative diagram

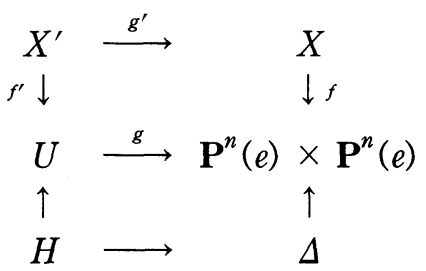

where the top square is cartesian, the vertical arrows of the bottom square are the canonical closed immersions, and the bottom horizontal arrow is an isomorphism. We shall need the following fact:

(2.3) In the hypotheses of Theorem (0.1), the variety $X^{\prime}=X \times_{\mathbf{P}^{n}(e) \times \mathbf{P}^{n}(e)} U$ is $(d+1)$-connected.

Indeed, since $X$ is $d$-connected, by Proposition (1.2) we can reorder the irre- 
ducible components $X_{1}, \ldots, X_{m}$ of $X$ (possibly with repetitions, by increaing $m$ if necessary) so that $\operatorname{dim}\left(X_{i-1} \cap X_{i}\right) \geq d$ for every $i=2, \ldots, m$. Set $X_{i}^{\prime}:=$ $g^{\prime^{-1}}\left(X_{i}\right), i=1, \ldots, m$. Observe that every closed fibre of $g$ (and hence also of $g^{\prime}$ ) is irreducible. Indeed, every closed fibre of $g$ is in fact isomorphic to $\mathbf{G}_{m}$. It follows that $X_{i}^{\prime}$ is irreducible for every $i=1, \ldots, m$, and in particular, $X_{1}^{\prime}, \ldots, X_{m}^{\prime}$ are the irreducible components of $X^{\prime}$. Moreover, since $\operatorname{dim}\left(X_{i-1} \cap X_{i}\right) \geq d$ we get that $\operatorname{dim}\left(X_{i-1}^{\prime} \cap X_{\imath}^{\prime}\right) \geq d+1$ for every $i=1, \ldots, m$. This proves the claim of (2.3) via Proposition (1.2).

(2.4) Now we can easily prove the first part of Theorem (0.1). By (2.3) and Proposition (2.1) (applied to the finite morphism $f^{\prime}: X^{\prime} \rightarrow U \subset \mathbf{P}^{2 n+1}(e, e)$ and $L:=H$, with $r=n+1 \leq d+1$ we infer that $f^{\prime-1}(H)$ is $(d-n)$-connected. On the other hand, since $f^{-1}(\Delta) \cong f^{\prime-1}(H)$ we get the first part of Theorem (0.1).

(2.5) Now we proceed to prove the second part of Theorem (0.1). I claim that one can reduce oneself to the case when $X$ is irreducible of dimension $d+1$ such that $d \geq n$. Indeed, if $X_{1}, \ldots, X_{m}$ are the irreducible components of $X$ then $X_{1} \backslash W, \ldots, X_{m} \backslash W$ are the irreducible components of $X \backslash W$. Then the claim follows easily from Corollary (1.8).

So, from now on assume $X$ irreducible of dimension $d+1$. Set $X^{\prime}:=f(X)$. I claim that it is sufficient to prove the second part of Theorem $(0.1)$ for $X^{\prime}$, i.e. we may assume that $f$ is a closed embedding.

Indeed, let $W$ be a closed subscheme of $Y:=f^{-1}(\Delta)$, of dimension $<d-r$ and set $W^{\prime}:=f(W)$. Then clearly $\operatorname{dim}(W)=\operatorname{dim}\left(W^{\prime}\right)=\operatorname{dim}\left(f^{-1}\left(W^{\prime}\right)\right)$ (because $f$ is finite), and $W \subset f^{-1}\left(W^{\prime}\right)$. Assuming that $X^{\prime} \cap \Delta \backslash W^{\prime}$ is $\mathbf{G}_{3}$ in $X^{\prime} \backslash W^{\prime}$, by Theorem (1.4) we infer that $Y \backslash f^{-1}\left(W^{\prime}\right)$ is $\mathbf{G}_{3}$ in $X \backslash f^{-1}\left(W^{\prime}\right)$.

On the other hand, since $W \subset f^{-1}\left(W^{\prime}\right)$ we have a commutative diagram

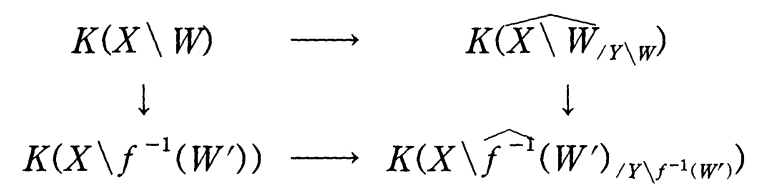

in which the vertical arrows are the restriction maps. The first vertical map is clearly an isomorphism, while the bottom horizontal map is an isomorphism because $Y \backslash f^{-1}\left(W^{\prime}\right)$ is $\mathbf{G}_{3}$ in $X \backslash f^{-1}\left(W^{\prime}\right)$. If we prove claim (2.6) below it will follow that the second vertical map is injective, and hence bijective because we just saw that the composition of it with the top horizontal map of the above commutative diagram is an isomorphism.

(2.6) The ring $K\left(\widehat{X \backslash W}_{/ Y \backslash W}\right)$ is a field.

To prove this we use Proposition (1.6). So, if $u: Z \rightarrow X$ is the normalization morphism it is sufficient to check that $u^{-1}(Y \backslash W)$ is connected. This follows from 
the first (i.e. the connectivity) part of Theorem (0.1) applied to the finite morphism $f \circ u: Z \rightarrow \mathbf{P}^{n}(e) \times \mathbf{P}^{n}(e)$ and the subscheme $u^{-1}(W)$ of $Z$, because

$$
u^{-1}(Y \backslash W)=(f \circ u)^{-1}(\Delta) \backslash u^{-1}(W) .
$$

(2.7) Summing up, to prove the second part of Theorem (0.1), we may assume that $f$ is a closed embedding and that $X$ is irreducible. In other words, from now on $X$ is an irreducible closed subscheme of $\mathbf{P}^{n}(e) \times \mathbf{P}^{n}(e)$.

According to the construction and notation of (2.2), set $U_{X}:=g^{-1}(X) \subset U$, and denote by $Z$ the closure of $U_{X}$ in $\mathbf{P}^{n}(e, e)$. Denote by $g^{\prime}: U_{X} \rightarrow X$ the restriction of $g$ to $U_{X}$. Then $g^{\prime}$ can be also considered as a rational map $g^{\prime}: Z \rightarrow X$ which is defined precisely in the open subset $U_{X}$ of $Z$.

Let $h: X_{1} \rightarrow Z$ be a proper morphism with the following properties:

$h$ is birational and the restriction $h / h^{-1}\left(U_{X}\right)$ defines an isomorphism between $h^{-1}\left(U_{X}\right)$ and $U_{X}$;

- the composition $f:=g^{\prime} \circ h: X_{1} \rightarrow X$ is a proper morphism (in particular, $f$ is everywhere defined).

The existence of $\left(X_{1}, h\right)$ is obvious because one can take as $X_{1}$ the closure of the graph $\Gamma_{g^{\prime}} \subset U_{X} \times X$ in $Z \times X$, and as $h$ the restriction to $X_{1}$ of the projection $Z \times X \rightarrow Z$. Then the restriction to $X_{1}$ of the projection $Z \times X \rightarrow X$ is a proper surjective morphism $f\left(=g^{\prime} \circ h\right)$, so that it makes sense to speak about the field extension $f^{*}: K(X)=K(X \backslash W) \rightarrow K\left(X_{1} \backslash f^{-1}(W)\right)$.

Applying Theorem (1.4) to the morphism $f: X_{1} \backslash f^{-1}(W) \rightarrow X \backslash W$, we get

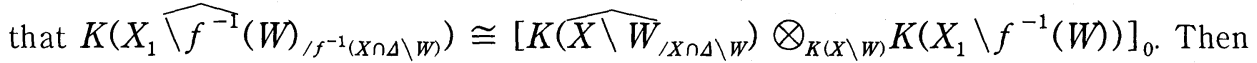
the key point of the proof of the second part of Theorem (0.1) is the following claim:

(2.8) The canonical map $K\left(X_{1} \backslash f^{-1}(W)\right) \rightarrow K\left(X_{1} \widehat{\backslash f^{-1}}(W)_{/ f^{-1}(X \cap \Delta \backslash W)}\right)$ is an isomorphism.

Accepting the claim (2.8) for the moment, we see that via the above isomorphism, the claim obviously implies the following fact:

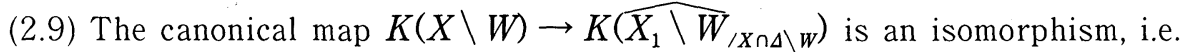
$X \cap \Delta \backslash W$ is $\mathbf{G}_{3}$ in $X \backslash W$. Therefore, in view of the reduction made at (2.5) we proved the last part of Theorem (0.1) modulo the claim (2.8).

(2.10) Now we proceed to the proof the claim of (2.8). Since by (2.2) $g / H$ defines an isomorphism $H \cong \Delta$ then $g^{\prime} / U_{X} \cap H$ defines an isomorphism $Z \cap H=$ $U_{X} \cap H \cong X \cap \Delta$. By the construction of $h$ the subvariety $Y:=h^{-1}(Z \cap H)$ is isomorphic to $Z \cap H \cong X \cap \Delta$ via $f / Y$. Since $Y \backslash f^{-1}(W) \subset f^{-1}(X \cap \Delta \backslash W)$ we get the canonical morphisms 


$$
\left.X_{1} \widehat{f^{-1}}(W)_{/ Y \backslash f^{-1}(W)}\right) \rightarrow X_{1} \widehat{{ }_{f^{-1}}^{-1}(W)_{/ f^{-1}(X \cap \Delta \backslash W)} \rightarrow X_{1} \backslash f^{-1}(W)},
$$

which yield the homomorphisms of $k$-algebras

$$
\begin{aligned}
K\left(X_{1} \backslash f^{-1}(W)\right) \stackrel{a}{\longrightarrow} & K\left(X_{1} \widehat{f^{-1}}(W)_{/ f^{-1}(X \cap \Delta \backslash W)}\right) \\
& K\left(X_{1} \widehat{\downarrow^{-1}}(W)_{/ Y \backslash f^{-1}(W)}\right) .
\end{aligned}
$$

Now, claim (2.8) follows from the following two claims:

(2.8.1) The composition $b \circ a$ is an isomorphism.

(2.8.2) $K\left(X_{1} \widehat{f^{-1}}(W)_{/ f^{-1}(X \cap \Delta \backslash W)}\right)$ is a field.

Indeed, by (2.8.2) the map $b$ is injective, and whence by (2.8.1), an isomorphism. By (2.8.1) again the map $a$ is an isomorphism.

It remains therefore to prove (2.8.1) and (2.8.2).

To prove (2.8.1) observe that since $H \subset U$ and $h / h^{-1}\left(U_{X}\right): h^{-1}\left(U_{X}\right) \rightarrow U_{X}$ $=Z \cap U$ is an isomorphism, we get

$$
\begin{aligned}
& K\left(X_{1} \backslash f^{-1}(W)\right) \cong K\left(Z \backslash h\left(f^{-1}(W) \cap Y\right)\right) \text {, and } \\
& \left.K\left(X_{1} \widehat{\backslash f^{-1}}(W)_{/ Y \backslash f^{-1}(W)}\right) \cong K\left(Z \backslash \widehat{h\left(f^{-1}\right.}(W)\right)_{/ Z \cap H \backslash h\left(f^{-1}(W) \cap Y\right)}\right) \text {. }
\end{aligned}
$$

So, we are reduced to prove that the canonical map

$$
K\left(Z \backslash W_{1}\right) \rightarrow K\left(\widehat{Z \backslash V}_{/ Z \cap H \backslash V}\right)
$$

is an isomorphism, where $V:=h\left(f^{-1}(W) \cap Y\right)$ is a closed subscheme of $Z \cap H$ such that $\operatorname{dim}(V)=\operatorname{dim}(W)<\operatorname{dim}(X)-n-1=\operatorname{dim}(Z)-(n+1)-1$, and $\operatorname{codim}_{\mathrm{P}^{2 n+1}(e, e)}(H)=n+1$. (Recall that $H=V_{+}\left(T_{0}-U_{0}, \ldots, T_{n}-U_{n}\right)$.) This will follow from the following more general assertion:

(2.10.1) For every closed irreducible subvariety $Z$ of $\mathbf{P}^{m}(e)=\operatorname{Proj}\left(k\left[T_{0}, \ldots\right.\right.$, $T_{m}$ ) (with $\operatorname{deg}\left(T_{i}\right)=e_{i}, i=0,1, \ldots, m$ ), for every subscheme $H$ of the form $H=V_{+}\left(T_{i_{1}}, \ldots, T_{i_{r}}\right)$, and for every closed subscheme $V$ of $Z \cap H$ such that $\operatorname{dim}(V)<\operatorname{dim}(Z)-r-1$, then $Z \cap H \backslash V$ is $\mathbf{G}_{3}$ in $Z \backslash V$.

To do this, consider the usual projective space $\mathbf{P}^{n}=\operatorname{Proj}\left(k\left[U_{0}, \ldots, U_{m}\right]\right)$, $\operatorname{deg}\left(U_{\imath}\right)=1, i=0,1, \ldots, m$. Consider the homomorphism of graded $k$-algebras

$$
\varphi: k\left[T_{0}, \ldots, T_{m}\right] \rightarrow k\left[U_{0}, \ldots, U_{m}\right]
$$

defined by $\varphi\left(T_{i}\right)=U_{i}^{e_{i}}, i=0,1, \ldots, m$. Then the morphism

$$
u:=\operatorname{Proj}(\varphi): P^{\prime}:=\mathbf{P}^{n} \rightarrow P:=\mathbf{P}^{n}(e)
$$

is finite and surjective.

(2.10.2) I claim that for every closed subvariety $Z$ of $P$, then $u^{-1}(Z)_{\text {red }}$ is 
$d$-connected.

Indeed, consider the morphism $f:=u \times i: P^{\prime} \times Z \rightarrow P \times P$, where $i: Z \rightarrow$ $P$ is the embedding of $Z$ in $P$. Since $Z$ is $d$-connected, $P^{\prime} \times Z$ is $(n+d)$ connected (as is easy to see). Then by the first part of Theorem (0.1) (already proved), $f^{-1}(\Delta) \cong u^{-1}(Z)$ is $d$-connected, as claimed.

In particular, coming back to (2.10.1), since $Z$ is irreducible, by (2.10.2), $Z^{\prime}:=u^{-1}(Z)$ is $(\operatorname{dim}(Z)-1)$-connected. If we set $H^{\prime}:=u^{-1}(H)_{\text {red }}$ then $H^{\prime}$ is the linear subspace of $\mathbf{P}^{n}$ given by $U_{i_{1}}=\cdots=U_{i_{r}}=0$. Thus, if $A:=Z \cap H$ then $u^{-1}(A)_{\text {red }}=Z^{\prime} \cap H^{\prime}$. By the result of Faltings in the form of Corollary (1.9) we infer that the natural map

$$
K\left(Z^{\prime} \backslash u^{-1}(V)\right) \rightarrow K\left(Z^{\prime} \widehat{u^{-1}}(V)_{/ u^{-1}(A \backslash V)}\right)
$$

is an isomorphism. On the other hand, the latter ring is by Theorem (1.4) (which can be applied because the fact that $Z^{\prime}$ is $(\operatorname{dim}(Z)-1)$-connected implies that every irreducible component of $Z^{\prime}$ dominates $Z$ ) canonically isomorphic to

$$
\left[K\left(\widehat{Z \backslash V}_{/ A \backslash V}\right) \otimes_{K(Z \backslash V)} K\left(Z^{\prime} \backslash u^{-1}(V)\right)\right]_{0} .
$$

These two isomorphisms imply (2.10.1). In this way (2.8.1) is proved.

(2.11) Now we shall prove (2.8.2). Let $v: X_{1}^{\prime} \rightarrow X_{1}$ be the normalization of $X_{1}$, and set $f^{\prime}:=f \circ v: X_{1}^{\prime} \rightarrow X \subset \mathbf{P}^{n}(e) \times \mathbf{P}^{n}(e)$. Using Proposition (1.6), all we have to check is that $v^{-1}\left(f^{-1}(X \cap \Delta \backslash W)\right)=f^{\prime-1}(\Delta \backslash W)$ is connected. Let $X_{1}^{\prime}$ $\stackrel{v^{\prime}}{\rightarrow} T \stackrel{v^{\prime \prime}}{\rightarrow} X \subset \mathbf{P}^{n}(e) \times \mathbf{P}^{n}(e)$ be the Stein factorization of $f^{\prime}$, i.e. $f=v^{\prime \prime} \circ v^{\prime}$, where $v^{\prime}$ has connected fibres, and $v^{\prime \prime}$ is a finite morphism. By the first part of Theorem (0.1) (already proved), $v^{\prime \prime}(\Delta \backslash W)$ is connected because $\operatorname{dim}\left(v^{\prime \prime}-1(W)\right)=$ $\operatorname{dim}(W)<\operatorname{dim}(X)-n-1$, by hypotheses, and hence $v^{\prime^{-1}}\left(v^{\prime \prime}(\Delta \backslash W)\right)=$ $f^{\prime-1}(\Delta \backslash W)$ is also connected because $v^{\prime}$ has connected fibres. Thus (2.8.2) is proved.

In this way the proof of Theorem (0.1) is complete.

Remark. It is well known that at least in characteristic zero, $\mathbf{P}^{n}(e)$ appears as the quotient of $\mathbf{P}^{n}$ by a finite group $G$ (which is the product of the cyclic groups of order $e_{i}, i=0, \ldots, n$ (see [3])). Then one may ask whether Theorem (0.1) holds true for every quotient $P^{\prime}:=\mathbf{P}^{n} / G$ of $\mathbf{P}^{n}$ by a finite group $G$ of automorphisms of $\mathbf{P}^{n}$. The answer is no in general. Indeed. consider the action of the group $G$ of roots of order $n+2$ of $k$, such that $n \geq 3, n+2$ is prime and different from $\operatorname{char}(k)$, on $\mathbf{P}^{n}$ given by $g\left(t_{0}, t_{1}, \ldots, t_{n}\right)=\left(t_{0}, g t_{1}, g^{2} t_{2}, \ldots, g^{n} t_{n}\right)$. Then the Fermat's hypersurface $F$ of $\mathbf{P}^{n}$ given by $t_{0}^{n+2}+t_{1}^{n+2}+\cdots+t_{n}^{n+2}=0$ is $G$-invariant and $G$ acts freely on $F$. If we take $X:=F \times F$, and as $f: X \rightarrow P^{\prime} \times$ 
$P^{\prime}$ the composition

$$
F \times F \subset \mathbf{P}^{n} \times \mathbf{P}^{n} \stackrel{u \times u}{\longrightarrow} P^{\prime} \times P^{\prime},
$$

(with $u: \mathbf{P}^{n} \rightarrow P^{\prime}$ the canonical morphism), then $f^{-1}\left(\Delta_{P^{\prime}}\right)$ has $n+2$ connected components, although $X$ is $(2 n-3)$-connected $(X$ is irreducible of dimension $2 n-2$ ) and $\operatorname{dim}\left(P^{\prime}\right)=n \leq 2 n-3$, if $n \geq 3$ (see the proof of Theorem (3.5) below and the example following it, for details).

\section{Proofs of Theorems (0.2) and (0.3) and other consequences}

First we mention the following direct consequence of Theorem (0.1).

Corollary (3.1). Let $Y$ and $Z$ be two irreducible subvarieties of $\mathbf{P}^{n}(e)$ such that $\operatorname{dim}(Y)+\operatorname{dim}(Z) \geq n+1$. Then $Y \cap Z \backslash W$ is $\mathbf{G}_{3}$ in $Y \times Z \backslash W$ for every closed subscheme $W$ of $Y \cap Z \cong(Y \times Z) \cap \Delta$ such that $\operatorname{dim}(W)<\operatorname{dim}(Y)+\operatorname{dim}(Z)$ $-n-1$. In particular, if $Y$ is a closed irreducible subvariety of $\mathbf{P}^{n}(e)$ of dimension $>\frac{n}{2}$, then $\Delta_{Y} \backslash W$ is $\mathbf{G}_{3}$ in $Y \times Y \backslash W$ for every closed subscheme $W$ of the diagonal $\Delta_{Y}$ of $Y \times Y$ such that $\operatorname{dim}(W)<2 \operatorname{dim}(Y)-n-1$.

Proof. Apply Theorem (0.1) to the inclusion of $X:=Y \times Z$ in $\mathbf{P}^{n}(e) \times$ $\mathbf{P}^{n}(e)$.

Q.E.D.

(3.2) The last part of Corollary (3.1) is just the first part of Theorem (0.2). In particular, $\Delta_{Y}$ is $\mathbf{G}_{3}$ in $Y \times Y$ for every irreducible subvariety $Y$ of $\mathbf{P}^{n}(e)$ of dimension $>\frac{n}{2}$. This latter statement implies the second part of Theorem (0.2). The argument to do that is essentially due to Speiser (see [20], or also [15], pp. 200-201), at least in the case when $Y$ is smooth. Below I shall only indicate the necessary steps in order to make Speiser's proof of the implication " $\Delta_{Y} \mathbf{G}_{3}$ in $Y \times$ $Y$ implies $\operatorname{Lef}\left(Y \times Y, \Delta_{Y}\right)$ ” work in this more general situation.

First, exactly as in Speiser's proof (loc. cit), we can reduce ourselves to the case when the vector bundle $E$ is of rank one, i.e. a line bundle. In this case, using the irreducibility of $Y$, we may assume that $E$ is a subsheaf of the constant sheaf $K(Y \times Y)$ (see e.g. [14], II, Prop. 6.15). Then all we have to check are the following two claims;

(3.2.1) For every $x:=(y, y) \in \Delta_{Y}$ we have

$$
\mathfrak{O}_{\widehat{Y \times Y, x}} \cap K(Y \times Y)=\mathfrak{O}_{Y \times Y, x},
$$


where the intersection is taken in the total ring of fractions of $\mathfrak{O}_{\widehat{Y \times Y}, x}$.

Using (3.2.1), the $\mathbf{G}_{3}$ property of $\left(Y \times Y, \Delta_{Y}\right)$, and the arguments given in [15], pp. 200-201, we infer that every section of $H^{0}(\widehat{Y \times Y}, \hat{E})$ comes from a section of $E$ defined in a suitable open neighbourhood $U$ of $\Delta_{Y}$ in $X \times Y$. This latter section of $E$ extends to the whole $Y \times Y$ by using the $\mathbf{S}_{2}$ property of $Y$ (which implies the $\mathbf{S}_{2}$ property of $\left.Y \times Y\right)$ and the following:

(3.2.2) $\operatorname{Codim}_{Y \times Y}(Y \times Y \backslash U) \geq 2$.

We first prove (3.2.1). In the case when $Y$ is normal a proof can be found in [20], pp, 16-17 (see also [15], p. 209, Ex. 4.12). However, as G. Chiriacescu pointed out, (3.2.1) comes from the following general statement. If $A \rightarrow B$ is a flat homomorphism of local rings, then $A=B \cap[A]_{0}$, where the intersection is taken in the total ring of fractions $[B]_{0}$ of $B$. To prove this statement, let $b=\frac{u}{v} \in B$, with $u, v \in A$, and $v$ a non-zero divisor of $A$. If $v$ is invertible there is nothing to prove. If $v$ belongs to the maximal ideal of $A$ then $u=b v \in B v \cap A=A v$ (the latter equality holds because the map $A \rightarrow B$ is faithfully flat). It follows that $b \in A$, as desired.

Now we prove (3.2.2). Set $X=Y \times Y$. Since $\operatorname{dim}(Y)>\frac{n}{2}$ then $X$ is a closed subvariety of $\mathbf{P}^{n}(e) \times \mathbf{P}^{n}(e)$ of dimension $>n$. Then the claim. follows from the construction of the proof of Theorem (0.1), because if $D$ were a hypersurface of $X$ which does not intersect $\Delta_{Y}=\Delta \cap X$ then (in the notations of the proof of Theorem (0.1)) $g^{\prime^{-1}}(D)$ would be a hypersurface of $U_{X}$ which does not intersect $U_{X} \cap H\left(U_{X} \cap H \subset g^{\prime-1}\left(\Delta_{Y}\right)\right)$, where $g^{\prime}=g / U_{X}: U_{X} \rightarrow X$ is a morphism whose closed fibres are all isomorphic to $\mathbf{G}_{m}$. Taking the closure $Z$ of $U_{X}$ in $\mathbf{P}^{2 n+1}(e, e)$, and the closure $D^{\prime}$ of $g^{\prime-1}(D)$ we would get a hypersurface of $Z$ which does not intersect $Z \cap H$. But recalling that $Z$ is a closed irreducible subvariety of $\mathbf{P}^{2 n+1}(e, e)$ of dimension $\geq n+2$ and $H=V_{+}\left(T_{0}-U_{0}, \ldots, T_{n}-U_{n}\right)$, this fact is impossible. Thus (3.2.2) is proved.

This finishes the proof of Theorem (0.2).

Q.E.D.

(3.3) Theorem (0.3) is a direct consequence of Theorems (0.2) and (1.11).

(3.4) Now consider the following situation:

- $Y$ a smooth projective subvariety of $\mathbf{P}^{n}(e)$ of dimension $>\frac{n}{2}$.

- $G$ a finite abelian group of order $d$ acting freely on $Y$ such that $d \geq 2$, and if $\operatorname{char}(k)>0$, then $d$ is prime to $\operatorname{char}(k)$. 
- Denote by $X$ the quotient $Y / G$.

Then we prove:

THEOREM (3.5). In the situation of (3.4) we have

$$
[K(\widehat{X \times X}): K(X \times X)]=d,
$$

where $K(\widehat{X \times X})$ is the field of formal rational functions of $X \times X$ along the diagonal $\Delta_{X}$. In particular, $\Delta_{X}$ is $\mathbf{G}_{2}$ (but not $\mathbf{G}_{3}$ ) in $X \times X$ (in the terminology of [16], or also [15]).

Proof. Denote by $u: Y \rightarrow X$ the canonical étale morphism. Then

$$
(u \times u)^{-1}\left(\Delta_{X}\right)=(G \times G) \Delta_{Y},
$$

where $G \times G$ acts on $Y \times Y$ in the canonical way.

A simple computation shows that if $g, g^{\prime}, h, h^{\prime} \in G$ then

$$
(g, g h) \Delta_{Y} \cap\left(g^{\prime}, g^{\prime} h^{\prime}\right) \Delta_{Y} \neq \emptyset \text { iff } h=h^{\prime} \text { iff }(g, g h) \Delta_{Y}=\left(g^{\prime}, g^{\prime} h^{\prime}\right) \Delta_{Y} .
$$

This shows that

$$
(u \times u)^{-1}\left(\Delta_{X}\right)=\Delta_{1} \cup \cdots \cup \Delta_{d},
$$

where $\Delta_{\imath}=\left(e, h_{i}\right) \Delta_{Y}, i=1, \ldots, d$, with $\left\{h_{1}=e, \ldots, h_{d}\right\}$ is a full system of representatives for the quotient group $(G \times G) / \Delta_{G}$ and $e$ the unity of $G$ (and in particular, $\Delta_{1}=\Delta_{Y}$ ). It follows that $\Delta_{i} \cap \Delta_{j}=\emptyset$ if $i \neq j$, and that for any $i$ and $j$ there is an element $\left(e, h_{i j}\right) \in G \times G$ such that $\left(e, h_{i j}\right) \Delta_{\imath}=\Delta_{j}$. From (3.5.1) it follows

$$
K\left(\widehat{Y \times Y}_{/(u \times u)^{-1}\left(\Delta_{X}\right)}\right)=\Pi_{i} K\left(\widehat{Y \times Y}_{/ \Delta_{i}}\right) \cong \Pi K\left(\widehat{Y \times Y}_{\Delta_{Y}}\right) .
$$

(The last product of (3.5.2) has $d$ factors). On the other hand, by Theorem (1.4) we have

$$
K\left(\widehat{Y \times Y}_{/(u \times u)^{-1}\left(\Delta_{Y}\right)}\right) \cong\left[K\left(\widehat{X \times X_{\Delta_{X}}}\right) \otimes_{K(X \times X)} K(Y \times Y)\right]_{0} .
$$

By Corollary (3.1) we have $K\left(\widehat{Y \times Y}_{/_{Y}}\right) \cong K(Y \times Y)$, and therefore (3.5.2) and (3.5.3) yield:

$$
K\left({\widehat{X \times X_{/ \Delta_{X}}}} \otimes_{K(X \times X)} K(Y \times Y) \cong \Pi K(Y \times Y)(d \text { times }) .\right.
$$

Here we have used the fact that the total ring of fractions of a product of fields coincides to that product itself. Finally, by Proposition (1.6), $K\left(\overline{X \times X}_{/_{X}}\right)$ is a field, and hence by (3.5.4) one concludes the proof of Theorem (3.5).

Q.E.D. 
EXAmple. If in Theorem (3.5) we take as $Y$ the Fermat's surface of equation $T_{0}^{5}+T_{1}^{5}+T_{2}^{5}+T_{3}^{5}=0$ with the action of the multiplicative group $G$ of roots of 1 order $5(\operatorname{char}(k) \neq 5)$ acting by

$$
g\left(t_{0}, t_{1}, t_{2}, t_{3}\right)=\left(t_{0}, g t_{1}, g^{2} t_{2}, g^{3} t_{3}\right), g \in G,
$$

then we get that the Godeaux surface $X=X / G$ has the property that $K(\widehat{X \times X})$ is a field extension of degree 5 of $K(X \times X)$. In particular, $\Delta_{X}$ is $\mathbf{G}_{2}$ (but not $\mathbf{G}_{3}$ ) in $X \times X$.

\section{Appendix}

In this appendix we give a proof of Theorem (1.3) of Grothendieck. Assume first that $X$ is irreducible of dimension $n>r$, and set $P:=\operatorname{Proj}(S)$ and $Y:=$ $f^{-1}\left(V_{+}\left(t_{1}, \ldots, t_{r}\right)\right)$. Since $P \backslash V_{+}\left(t_{1}, \ldots, t_{r}\right)=D_{+}\left(t_{1}\right) \cup \cdots \cup D_{+}\left(t_{r}\right), f$ is finite and $D_{+}\left(t_{i}\right)$ is affine, it follows that $X \backslash Y$ is the union of the affine open subsets $f^{-1}\left(D_{+}\left(t_{i}\right)\right), i=1, \ldots, r$. If $Z$ is an algebraic variety, consider Hartshorne's cohomological dimension $\operatorname{cd}(Z)$ of $Z$ defined by $\operatorname{cd}(Z):=\max \left\{m \geq 0 / H^{m}(Z, F)\right.$ $\neq 0$ for some coherent sheaf $F \in \operatorname{Coh}(Z)\}$. It follows that $\operatorname{cd}(X \backslash Y) \leq r-1$ $\leq n-1$ (resp. $\operatorname{cd}(X \backslash Y) \leq n-2$ if $r \leq n-1)$. Now, we have the following:

Proposition (Hartshorne-Lichtenbaum). In the above situation, the inequqlity $c d(X \backslash Y) \leq n-1$ (resp. $c d(X \backslash Y) \leq n-2)$ implies $Y \neq \emptyset$ (resp. $Y$ connected $)$.

Proof. The variety $X$ is projective because $f$ is a finite morphism and $P$ is projective. The first assertion follows from the so-called Hartshorne-Lichtenbaum theorem (see [11], [17], or [15]), asserting in this case that $\operatorname{cd}(X \backslash Y)=n$ if and only if $Y=\emptyset$. Assume therefore ( $X$ irreducible and) $\operatorname{cd}(X \backslash Y) \leq n-2$, and $Y$ disconnected, with $Y=Y_{1} \cup Y_{2}, Y_{1}, Y_{2}$ non-empty closed subsets of $Y$ such that $Y_{1} \cap Y_{2}=\emptyset$. Because $X$ is projective of dimension $n$, there is an invertible sheaf $L$ on $X$ such that $H^{n}(X, L) \neq 0$ (just take any sufficiently negative power of an ample line bundle on $X$ ).

In the exact sequence

$$
H_{Y_{i}}^{n}(X, L) \rightarrow H^{n}(X, L) \rightarrow H^{n}\left(X \backslash Y_{\imath}, L\right)
$$

the last space is zero by Hartshorne-Lichtenbaum theorem because $Y_{i} \neq \emptyset$ for $i=1,2$. It follows that $h_{Y_{i}}^{n}(X, L) \geq h^{n}(X, L)$ for $i=1,2$. Moreover, $H_{Y}^{n}(X, L)$ $\cong H_{Y_{i}}^{n}(X, L) \oplus H_{Y_{2}}^{n}(X, L)$, and hence, $h_{Y}^{n}(X, L) \geq 2 h^{n}(X, L)$.

On the other hand, in the exact sequence 


$$
H^{n-1}(X \backslash Y, L) \rightarrow H_{Y}^{n}(X, L) \rightarrow H^{n}(X \backslash L) \rightarrow H^{n}(X \backslash Y, L)
$$

the extreme spaces are zero by $\operatorname{cd}(X \backslash Y) \leq n-2$ whence $h_{Y}^{n}(X, L)=h^{n}(X, L)$, contradicting the previous inequality because $h^{n}(X, L) \geq 1$.

Q.E.D.

Remark. Consider the Serre map of $P=\operatorname{Proj}(S)$

$$
a: S \rightarrow \bigoplus_{a \geq 0} H^{0}\left(P, \mathcal{O}_{P}(a)\right),
$$

and for $t_{i} \in S_{a_{i}}, i=1, \ldots, r$, set $s_{i}:=\alpha\left(t_{i}\right) \in H^{0}\left(P, \mathcal{O}_{P}\left(a_{i}\right)\right)$. Then for a point $p \in P$ the following statement holds (even if $S$ is not generated by $S_{1}$ ) $: p \in$ $D_{+}\left(t_{i}\right)$ if and only if $s_{i}(p) \neq 0$. (Recall that $s_{i}(p) \neq 0$ means that $\left(s_{i}\right)_{p} \notin$ $m_{p} \mathfrak{O}_{P}\left(a_{i}\right)_{p}$, where $m_{p}$ is the maximal ideal of $\left.\mathscr{O}_{p}\right)$. With this observation, the proof of the above lemma yields in fact the following more general assertion. Assume that for every $i=1, \ldots, r$ we are given a finitely generated graded $k$-algebra $S^{2}$, a homogeneous element $t_{i} \in\left(S^{i}\right)_{+}$and a finite morphism $f_{i}: X \rightarrow \operatorname{Proj}\left(S^{i}\right)$. Set $s_{i}:=f_{i}^{*}\left(\alpha_{i}\left(t_{i}\right)\right)$ (where $\alpha_{i}$ is the Serre map of $P_{i}:=\operatorname{Proj}\left(S^{2}\right)$ ). Then the zero locus of the section $\left(s_{1}, \ldots, s_{r}\right)$ of $\bigoplus_{i} H^{0}\left(X, f_{i}^{*}\left(\mathscr{O}_{P_{i}}\left(\operatorname{deg}\left(t_{i}\right)\right)\right)\right.$ is $(d-r)$ connected if $X$ is $d$-connected and $d \geq r$.

Let us proceed now to the proof of Theorem (1.3). Let $X_{1}, \ldots, X_{m}$ be the irreducible components of $X$. Since $X$ is $d$-connected, $\operatorname{dim}\left(X_{i}\right)>d+1$ for every $i=$ $1, \ldots, m$. But by what we have proved before $Y_{i}:=Y \cap X_{i}$ is connected (and non-empty) for every $i=1, \ldots, m$, where, as above, $Y:=f^{-1}\left(V_{+}\left(t_{1}, \ldots, t_{r}\right)\right.$. Since $X$ is $d$-connected, by Proposition (1.2), we can reorder the components $X_{1}, \ldots, X_{m}$ (possibly with repetitions) so that for every $i=2, \ldots, m$ there is an irreducible component $Z_{i}$ of $X_{i-1} \cap X_{i}$ of dimension $\geq d$. Applying the first part of the above lemma to $Z_{i}$ we infer that $Y_{i-1} \cap Z_{i}=Y_{i} \cap Z_{i}=Y \cap Z_{i}$ is not empty. This implies that $Y_{i-1} \cap Y_{i} \neq \varnothing$ for every $i=2, \ldots, m$. In particular, $Y$ is connected.

We prove now that $Y$ is $(d-r)$-connected if $X$ is $d$-connected. Let $X \subset \mathbf{P}^{N}$ be an arbitrary projective embedding of $X$, and let $A$ be a general linear subspace of $\mathbf{P}^{N}$ of dimension $N+r-d$. Because every irreducible of $X$ is of dimension $\geq d+1$ and because $Y$ is locally given by $r$ equations in $X$, every irreducible component $Z$ of $Y$ is of dimension $\geq d+1-r$. It follows that $\operatorname{dim}(Z \cap A)=$ $\operatorname{dim}(Z)+\operatorname{dim}(A)-N \geq(d+1-r)+(N+r-d)-N=1$, and in particular, $A$ meets every irreducible component of $Y$.

Set $Y^{\prime}:=Y \cap A$. If $A$ is defined by linear equations $t_{r+1}=\ldots t_{d}=0$ in $\mathbf{P}^{N}$ then $Y^{\prime}$ is just the zero locus of the section $\left(t_{r+1} / X, \ldots, t_{d} / X, s_{1}, \ldots, s_{r}\right)$ of the sheaf $(d-r) \mathscr{O}_{X}(1) \oplus f^{*}\left(\mathscr{O}_{P}\left(a_{r}\right)\right) \oplus \cdots \oplus f^{*}\left(\mathscr{O}_{P}\left(a_{r}\right)\right)$, where for $i=1, \ldots, r$, 
$t_{\imath} \in S_{a_{i}}, s_{i}:=f^{*}\left(\alpha\left(t_{i}\right)\right)$, and $a: S \rightarrow \bigoplus_{j} H^{0}\left(P, \mathscr{O}_{P}(j)\right)$ is the Serre map of $P=$ $\operatorname{Proj}(S)$. By the above lemma and the remark following it, $Y^{\prime}$ is connected.

Now, assume that $Y$ is not $(d-r)$-connected, i.e. there is a closed subscheme $W$ of $Y$ of dimension $<d-r$ such that $Y \backslash W$ is disconnected. Since $A$ is general, $\operatorname{dim}(W \cap A)=\operatorname{dim}(W)+\operatorname{dim}(A)-N<(d-r)+(N+r-d)-N=0$, or else, $A$ does not meet $W$. Moreover, since $A$ meets every irreducible component of $Y$, from the fact that $Y \backslash W$ is disconnected, it follows that $Y^{\prime}=Y \cap A=(Y \backslash W)$ $\cap A$ is also disconnected, a contradiction.

Q.E.D.

\section{REFERENCES}

[1] L. Bădescu and E. Ballico, Formal rational functions along zero loci of sections of ample vector bundles, Rev. Roumaine Math. Pures Appl., 38 (1993), 609-630.

[2] P. Deligne, Le groupe fondamental du complément d'une courbe plane n'ayant que des points doubles ordinaires, Séminaire Bourbaki No 543, Paris, Novembre 1979.

[ 3 ] I. Dolgachev, Weighted projective varieties, Lecture Notes in Math., vol. 956, Springer Verlag, Berlin-Heidelberg-New York, 1982, pp. 34-71.

[4] G. Faltings, A contribution to the theory of formal meromorphic functions, Nagoya Math. J., 77 (1980), 99-106.

[5] W. Fulton and J. Hansen, A connectedness theorem for projective varieties with applications to intersections and singularities of mappings, Annals of Math., 110 (1979), 159-166.

[6] W. Fulton and R. Lazarsfeld, Connectivity and its applications in algebraic geometry, Lecture Notes in Math., vol. 862, Springer Verlag, Berlin-Heidelberg-New York, 1981, pp. 26-92.

[7] D. Gieseker, Flat bundles and the fundamental group in non-zero characteristics, Annali Sc. Norm. Pisa, 2 (1975), 1-31.

[8] A. Grothendieck, Revêtements étales et groupe fondamental, Lecture Notes in Math., vol. 224, Springer Verlag, Berlin-Heidelberg-New York, 1971.

[9] - Cohomologie locale des faisceaux cohérents et théorèmes de Lefschetz locaux et globaux, North Holland, Amsterdam, 1968.

[10] - Dix éxposés sur la cohomologie des schémas, North Holland, Amsterdam, 1968.

[11] - Local cohomology, Lecture Notes in Math. vol. 41, Springer Verlag, Berlin-Heidelberg-New York, 1966.

[12] J. Hansen, Higher order singularities and morphisms to projective spaces, Proc. Amer. Math. Soc., 97 (1986), 226-232.

[13] R. Hartshorne, Complete intersections and connectedness, Amer. J. Math., 84 (1962), 497-508.

[14] - Algebraic Geometry, Springer Verlag, Berlin-Heidelberg-New York, 1977.

[15] - Ample subvarieties of algebraic varieties, Lecture Notes in Math., vol. 156, Springer Verlag, Berlin-Heidelberg-New York, 1970.

[16] H. Hironaka and H. Matsumura, Formal functions and formal embeddings, J. Math. Soc. Japan, 20 (1968), 52-82.

[17] S. Kleiman, On the vanishing of $H^{n}(X, F)$ for an $n$-dimensional variety, Proc. 
Amer. Math. Soc., 18 (1967), 940-944.

[18] A. Ogus, Local cohomological dimension of algebraic varieties, Annals of Math., $\mathbf{9 8}$ (1973), 327-365.

[19] J.-P. Serre, Algèbre locale, Multiplicités, Lecture Notes in Math., vol. 11, Springer Verlag, Berlin-Heidelberg-New York, 1965.

[20] R. Speiser, Cohomological dimension of abelian varieties, Amer. J. Math., 95 (1973), $1-34$.

[21] - Projective varieties of low codimension in characteristic $p>0$, Trans. Amer. Math. Soc., 240 (1978), 329-343.

[22] O. Zariski, Theory and applications of holomorphic functions on algebraic varieties over arbitrary ground fields, Memoirs of the American Math. Soc., vol. 5, Rhode Island, 1951.

Institute of Mathematics of the Romanian Academy

P.O. Box 1-764, Ro- 70700

Bucharest, Romania

and

University of Bucharest

Department of Mathematics

E-mail address: lbadescu@stoilow.imar.ro 